\title{
Determinação de limites superiores da zona de conforto térmico para codornas de corte aclimatizadas no Brasil de 22 a 35 dias de idade ${ }^{1}$
}

\author{
Determination of upper limits of the thermal comfort zone for quails acclimatized in \\ Brazil 22-35 days old
}

\author{
SOUSA, Marilú Santos ${ }^{2 *}$; TINÔCO, Ilda de Fátima Ferreira ${ }^{3}$; BARRETO, Sérgio Luiz \\ de Toledo ${ }^{3}$; AMARAL, Adriana Garcia do ${ }^{4}$; PIRES, Luanna Chácara ${ }^{5}$; FERREIRA, \\ Aloízio Soares $^{3}$
}

\footnotetext{
${ }^{1}$ Parte da tese de doutorado da primeira autora.

${ }^{2}$ Universidade Federal do Tocantins, Escola de Medicina Veterinária e Zootecnia, Araguaína, Tocantins, Brasil.

${ }^{3}$ Universidade Federal de Viçosa, Centro de Ciências Agrárias, Departamento de Engenharia Agrícola e Departamento de Zootecnia, Viçosa, Minas Gerais, Brasil.

${ }^{4}$ Universidade Federal do Mato Grosso, Instituto de Ciências Agrárias e Ambientais, Sinop, Mato Grosso, Brasil.

${ }^{5}$ Universidade Federal do Piauí, Campus Professora Cinobelina Elvas, Bom Jesus, Piauí, Brasil.

*Endereço para correspondência: mariluzoo@ hotmail.com
}

\section{RESUMO}

Objetivou-se nesta pesquisa determinar as faixas de conforto térmico, avaliadas em termos de temperatura e Índices de Temperatura de Globo Negro e Umidade (ITGU), adequadas à criação de codornas de corte na fase final de criação. Foram alojadas 300 codornas de corte, em diferentes ambientes térmicos. Cada grupo de 60 aves foi distribuído, aleatoriamente, em 03 câmaras climáticas, sendo 10 aves por unidade experimental (gaiola), totalizando 15 tratamentos. Os tratamentos foram: CP Conforto Preconizado (temperaturas de 26 e 25 ${ }^{\circ} \mathrm{C}$, respectivamente, para quarta e quinta semana de criação); QM - Calor Moderado (30 ${ }^{\circ} \mathrm{C}$ para a quarta e quinta semana de criação) e QS - Calor Severo $\left(33{ }^{\circ} \mathrm{C}\right.$ para a quarta e quinta semana de criação). Durante a quarta semana de criação das codornas (22 a 28 dias), o melhor valor de ITGU foi de 75,8 $\pm 0,9$ e para a quinta semana de criação (29 a 35 dias) ITGU de 75,3 $\pm 0,7$ (indicativos de conforto térmico para esta idade). Verificou-se que: codornas mantidas em estresse térmico (seja por frio ou calor) durante a fase inicial de criação não se recuperam ao final do ciclo de vida; mesmo quando criadas em condições de conforto térmico na fase inicial, após este período, quando mantidas em temperaturas de estresse seja por calor moderado ou severo crônicos, não conseguem se manter com a performance normal, demonstrando a necessidade de se garantir que o ambiente térmico dos aviários seja mantido adequado de acordo com as exigências para cada idade das codornas.

Palavras-chave: ambiência, Coturnix, desempenho

\section{SUMMARY}

The objective of this research was to determine the temperature comfort, evaluated in terms of temperature and indices Black Globe Temperature and Humidity (BGTH), appropriate to the creation of quails in the final phase. Were housed 300 quails in different thermal environments. Each group of 60 birds were distributed randomly into 03 chambers, with 10 birds per pen (cage), totaling 15 treatments. The treatments were: CP - Comfort Pressed (temperatures of 26 and $25{ }^{\circ} \mathrm{C}$, respectively, for fourth and fifth week of creation); $\mathrm{MH}$ - Moderate heat $\left(30{ }^{\circ} \mathrm{C}\right.$ for the fourth and fifth week of creation) and HS - Heat Severus $\left(33{ }^{\circ} \mathrm{C}\right.$ for the fourth and fifth week of creation). During the fourth week of creation quail (22-28 days), the best value of BGTH was $75.8 \pm 0.9$ and for the fifth week of creation (2935 days) BGTH $75.3 \pm 0,7$ (indicative of 
thermal comfort for this age). It was found that: quail maintained in thermal stress (either hot or cold) during the initial phase can not recover the end of the life cycle, even when reared under conditions of thermal comfort in the initial stage after this period, while kept at temperatures of heat stress is moderate or severe chronic, can not keep up with the normal performance, demonstrating the need to ensure that the thermal environment of poultry is kept in accordance with the appropriate requirements for each age of the quail.

Keywords: carcass, Coturnix, environment

\section{INTRODUÇÃO}

O ambiente ao qual as aves estão inseridas compreende todos os elementos físicos, químicos, biológicos, sociais e climáticos que influenciam o seu desenvolvimento e crescimento. Dentre estes, os elementos climáticos, componentes do ambiente térmico do animal, incluem a temperatura, a umidade relativa, movimentação do ar e radiação, sendo estes os mais relevantes, por exercerem ação direta e imediata sobre as respostas comportamentais, produtivas e reprodutivas dos animais (BAÊTA \& SOUZA, 2010).

Segundo Silva et al. (2012), o processo da manutenção da homeotermia, somente é eficiente quando a temperatura ambiental estiver dentro dos limites da termoneutralidade, sendo que as aves não se ajustam, perfeitamente, em extremos de temperatura, podendo, inclusive, ter a vida ameaçada. Dessa forma, é importante que as aves sejam alojadas em ambientes, onde seja possível o balanço térmico.

Segundo Oliveira et al. (2006), de uma maneira geral um ambiente é considerado confortável para aves adultas quando apresenta temperatura de $21^{\circ} \mathrm{C}$ e umidade relativa do ar de 57 a $69 \%$. Entretanto, dificilmente estes valores são encontrados em condições comerciais de produção, sobretudo no verão.

Diante do exposto objetivou-se nesta pesquisa determinar as faixas de conforto térmico, avaliadas em termos de temperatura e Índices de Temperatura de Globo Negro e Umidade (ITGU), realmente adequadas à criação de codornas de corte (Coturnix coturnix coturnix) em sua fase final de criação, que vai de 22 até 35 dias de vida, ocasião em que são mais vulneráveis às altas temperaturas. Objetivou-se, também, investigar se codornas de corte criadas em condições de conforto térmico na fase inicial de vida, são capazes de manter seu desempenho normal, caso venham a ser mantidas, na fase final de criação, em condições mais ou menos severas de estresse por calor. Ou, por outro lado: se aves criadas em níveis mais ou menos acentuados de estresse por frio ou por calor em sua fase inicial de vida, se tornam mais adaptadas e resistem melhor em sua fase final de vida, quando submetidas a ambientes de estresse por calor moderado ou agudo.

\section{MATERIAL E MÉTODOS}

O experimento foi realizado em três câmaras climáticas, com as seguintes dimensões: 2,5 x 3,5 x 2,5m, localizadas no Núcleo de Pesquisa em Ambiência e Engenharia de Sistemas Agroindustriais (AMBIAGRO), da Universidade Federal de Viçosa. A coleta dos dados foi realizada no período de janeiro à fevereiro de 2012, totalizando 2 semanas experimentais em campo (14 dias).

Cada câmara climática foi equipada com um aquecedor de ar de resistência elétrica, um condicionador de ar do tipo "split" quente / frio e um umidificador de ar. 
Este experimento foi conduzido a partir do $22^{\circ}$ até o $35^{\circ}$ dia de idade das codornas (ciclo final de produção). $\mathrm{Na}$ condução desta pesquisa, foram utilizadas 300 codornas de corte (Coturnix coturnix coturnix), de ambos os sexos, com 22 dias de vida, originárias de uma primeira fase experimental, na qual estes animais, em grupos distintos de 60 aves, já haviam sido alojados em diferentes temperaturas. Desta forma, um grupo de 60 aves tiveram o crescimento inicial (período de 1 a 21 dias de idade) conduzido em ambiente considerado confortável, um grupo de 60 aves em ambiente de estresse por calor moderado, 60 aves em ambiente de estresse por calor agudo, 60 aves em ambiente de estresse frio moderado e 60 aves em ambiente de estresse por frio agudo. Cada um destes grupos de 60 aves foram redistribuídos em grupos de 20 indivíduos os quais foram realocados, aleatoriamente, em três câmaras climáticas, totalizando 20 aves por tratamento, sendo 10 aves por unidade experimental, sendo 10 gaiolas por câmara, o que totalizou os 15 tratamentos (Tabela 1).

Tabela 1. Ambientes térmicos em função das temperaturas do ar ambiente no interior das câmaras climáticas durante a quarta e quinta semanas de vida das codornas

\begin{tabular}{|c|c|c|c|}
\hline $\begin{array}{l}\text { Ambientes } \\
\text { Térmicos }\end{array}$ & $\begin{array}{l}\text { Descrição de cada ambiente (F1: fase inicial de } \\
\text { criação - } 1 \text { a } 21 \text { dias; F2: fase de crescimento - } 22 \text { a } \\
35 \text { dias) }\end{array}$ & $\begin{array}{l}\text { Temperatura } 4^{\mathrm{a}} \\
\text { semana }\left({ }^{\circ} \mathrm{C}\right)\end{array}$ & $\begin{array}{l}\text { Temperatura } \\
5^{\mathrm{a}} \text { semana } \\
\left({ }^{\circ} \mathrm{C}\right)\end{array}$ \\
\hline $\mathrm{CF} 1 / \mathrm{CF} 2$ & $\begin{array}{c}\text { Conforto Preconizado F1 + Conforto Preconizado } \\
\text { F2 (CF1/CF2) }\end{array}$ & 26 & 25 \\
\hline $\mathrm{FMF} 1 / \mathrm{CF} 2$ & $\begin{array}{l}\text { Frio moderado F1+ Conforto Preconizado F2 } \\
\text { (FMF1/CF2) }\end{array}$ & 26 & 25 \\
\hline $\mathrm{FSF} 1 / \mathrm{CF} 2$ & $\begin{array}{c}\text { Frio severo F1+ Conforto Preconizado F2 } \\
\text { (FSF1/CF2) }\end{array}$ & 26 & 25 \\
\hline $\mathrm{QMF} 1 / \mathrm{CF} 2$ & $\begin{array}{c}\text { Calor moderado F1 + Conforto Preconizado F2 } \\
\text { (QMF1/CF2) }\end{array}$ & 26 & 25 \\
\hline QSF1/CF2 & $\begin{array}{c}\text { Calor severo F1 + Conforto Preconizado F2 } \\
\text { (QSF1/CF2) }\end{array}$ & 26 & 25 \\
\hline CF1/QMF2 & $\begin{array}{c}\text { Conforto Preconizado F1+ Calor moderado F2 } \\
\text { (CF1/QMF2) }\end{array}$ & 30 & 30 \\
\hline FMF1/QMF2 & $\begin{array}{c}\text { Frio moderado F1 + Calor moderado F2 } \\
\text { (FMF1/QMF2) }\end{array}$ & 30 & 30 \\
\hline FSF1/QMF2 & Frio severo F1+ Calor moderado F2 (FSF1/QMF2) & 30 & 30 \\
\hline QMF1/QMF2 & $\begin{array}{c}\text { Calor moderado F1 + Calor moderado F2 } \\
\text { (QMF1/QMF2) }\end{array}$ & 30 & 30 \\
\hline QSF1/QMF2 & $\begin{array}{c}\text { Calor severo F1 + Calor moderado F2 } \\
\text { (QSF1/QMF2) }\end{array}$ & 30 & 30 \\
\hline CF1/QSF2 & $\begin{array}{c}\text { Conforto Preconizado F1+ Calor severo F2 } \\
\text { (CF1/QSF2) }\end{array}$ & 33 & 33 \\
\hline FMF1/QSF2 & $\begin{array}{c}\text { Frio moderado F1 + Calor severo F2 } \\
\text { (FMF1/QSF2) }\end{array}$ & 33 & 33 \\
\hline FSF1/QSF2 & Frio severo F1 + Calor severo F2 (FSF1/QSF2) & 33 & 33 \\
\hline QMF1/QSF2 & $\begin{array}{c}\text { Calor moderado F1+ Calor severo F2 } \\
(\mathrm{QMF} 1 / \mathrm{QSF} 2)\end{array}$ & 33 & 33 \\
\hline QSF1/QSF2 & Calor severo F1 + Calor severo F2 (QSF1/QSF2) & 33 & 33 \\
\hline
\end{tabular}


A umidade relativa do ar no interior das câmaras climáticas, durante todo $\mathrm{o}$ período experimental para todos os tratamentos, foi de $55 \%( \pm 5 \%)$, por ser considerado um valor adequado à produção avícola (MEDEIROS et. al, 2005). Os valores de temperaturas e de umidade relativa foram mantidos constantes, ou seja, 24 horas diárias. Assim, a temperatura do ar, foi a única variável de interferência no desempenho produtivo das codornas.

Foi fornecido ração de crescimento, conforme preconizado por Silva \& Costa (2009). A composição e os valores nutricionais dos ingredientes utilizados na formulação das rações foram definidos segundo Rostagno et al. (2011), e todos os tratamentos receberam a mesma ração. Esta ração foi fornecida a vontade, de forma que os comedouros estivessem sempre abastecidos, sendo que este manejo era realizado nos horários de 8:00 e 16:00 horas.

O programa de luz adotado foi o contínuo, com uma hora de escuro e 23 horas de luz durante todo o período experimental.

Com base na premissa, de que as exigências térmicas das aves domésticas mudam de acordo com seu crescimento, foram definidas faixas de condições térmicas diferentes para cada uma das câmaras climáticas. Uma destas faixas foi definida como sendo a faixa de conforto térmico (preconizado pela literatura), conforme sugerido por Albino e Barreto (2003), as demais como sendo dois níveis de estresse por calor (severo \& moderado).

Desta forma, as codornas foram submetidas aos diferentes ambientes térmicos: CP - Conforto Preconizado (temperaturas de 26 e $25^{\circ} \mathrm{C}$, respectivamente, para quarta e quinta semana de criação); QM - Calor Moderado $\left(30^{\circ} \mathrm{C}\right.$ para a quarta e quinta semana de criação) e QS - Calor Severo $\left(33{ }^{\circ} \mathrm{C}\right.$ para a quarta e quinta semana de criação). As temperaturas obtidas em cada ambiente, foram comparadas quanto ao desempenho zootécnico (consumo de ração, peso corporal, ganho de peso e eficiência alimentar), como forma de identificar as faixas de conforto para as codornas de corte em cada uma das semanas da fase final de vida destas.

Para os dados relativos ao desempenho das codornas foram registrados, semanalmente, os dados de ganho de peso (GP), em gramas; consumo de ração (CR), em gramas; eficiência alimentar (obtido pela relação: EA= ganho de peso em $\mathrm{g} /$ consumo de ração em g) e viabilidade de criação (número de aves vivas dividido pelo total de aves multiplicado por 100 , ou seja, em \%).

$\mathrm{O}$ controlo do ambiente térmico, requeridos no interior de cada câmara climática, foram registrados diariamente por meio de dataloggers de medição de temperatura e umidade com resolução de $0,1{ }^{\circ} \mathrm{C}$. As coletas das variáveis ambientais foram feitas a cada cinco minutos. E, diante dos valores registrados, foi calculado o Índice de Temperatura de Globo Negro e Umidade (ITGU), para cada ambiente térmico, com base na equação de Buffington et al. (1981).

A ventilação higiênica aplicada no interior das câmaras climáticas foi feita através de 02 exaustores axiais, com acionamento automático, de forma a permitir 04 renovações de ar por hora durante todo o período experimental, o que proporcionou a manutenção da qualidade do ar em boas condições.

Os dados obtidos foram submetidos ao teste $\mathrm{F}$ da análise de variância e as médias foram comparadas pelo teste de Tukey ao nível de 5\% de probabilidade. Utilizou-se o Programa Sistema para Análises Estatísticas e Genética (UFV, 2007). 


\section{RESULTADOS E DISCUSSÃO}

Valores de temperatura e umidade relativa do ar ambiente, e respectivos valores para ITGU correspondentes para a fase de crescimento das codornas (quarta e quinta semana de vida) para os diferentes ambientes térmicos (Tabela 2).
Como observado, os valores de temperatura do ar e umidade relativa do ar mantiveram-se próximos aos valores propostos para cada condição térmicoambiental descrita na metodologia, indicando que o controle térmico ambiental das câmaras climáticas foi adequado.

Tabela 2. Médias e desvios padrões dos valores de temperatura do ar (Tar), umidade relativa do ar (UR) e índice temperatura de globo negro e umidade (ITGU) para cada condição climática avaliada no período de 22 a 35 dias de vida das codornas de corte

\begin{tabular}{|c|c|c|c|}
\hline Ambientes Térmicos & $\operatorname{Tar}\left({ }^{\circ} \mathrm{C}\right)$ & UR (\%) & ITGU \\
\hline \multicolumn{4}{|c|}{ Quarta Semana (22 a 28 dias) } \\
\hline Conforto Preconizado (CP) & $26,7 \pm 0,6$ & $60,2 \pm 6,4$ & $75,8 \pm 0,9$ \\
\hline Calor Moderado (QM) & $30,4 \pm 0,8$ & $59,3 \pm 4,0$ & $79,7 \pm 1,0$ \\
\hline Calor Severo (QS) & $33,0 \pm 0,8$ & $57,6 \pm 8,0$ & $80,8 \pm 1,1$ \\
\hline \multicolumn{4}{|c|}{ Quinta Semana (29 a 35 dias) } \\
\hline Conforto Preconizado (CP) & $25,6 \pm 0,6$ & $60,7 \pm 4,5$ & $75,3 \pm 0,7$ \\
\hline Calor Moderado (QM) & $30,5 \pm 0,4$ & $61,1 \pm 3,5$ & $79,9 \pm 0,5$ \\
\hline Calor Severo (QS) & $33,2 \pm 0,2$ & $61,0 \pm 6,4$ & $82,2 \pm 0,4$ \\
\hline
\end{tabular}

Ainda não existem, em literatura, dados específicos para valores de ITGU ideais para codornas destinadas a corte. Diante disso, os valores obtidos neste experimento foram comparados com aqueles já encontrados em literatura, para frangos de corte. Entende-se que, desta forma, dar-se-á início a um banco de dados interessante para análises futuras que possibilitem comprovar realmente o valor de conforto térmico para codornas de acordo com o ITGU. Teixeira (1983) admitiu valores de ITGU confortáveis para a criação de frangos de corte em torno de 65 a 77 a partir da terceira semana de vida das aves. Diante disto, e considerando este um parâmetro de referência, pode-se inferir que as codornas mantidas no tratamento $\mathrm{CP}$, estiveram em conforto, tanto para a quarta, como para a quinta semanas de vida destas.
Corroborando com estes dados, Furtado et al. (2006) assumiram que o ITGU com valor de até 77 não influencia no desempenho de aves adultas. Medeiros et al. (2005), afirma que valores de ITGU de 78 a 88 caracterizam ambiente quente para frangos de corte. Ainda, dentro desta premissa, Vaz et al. (2009), verificaram que o valor de ITGU de 82,9 na fase final de criação de frangos de corte, caracterizam ambiente de calor.

Desta forma, pode-se inferir, com base nos autores citados, que as condições de calor moderado e severo aplicadas neste experimento, estão acima do limite de conforto para as codornas de corte em sua fase final de criação.

Verifica-se que houve influência $(\mathrm{P} \leq 0,05)$ dos ambientes térmicos sobre o ganho de peso das aves. Entretanto, o consumo de ração, a conversão 
alimentar e a viabilidade das aves não foram influenciados $(\mathrm{P} \geq 0,05)$ pelos ambientes térmicos de criação adotados (Tabela 3).

Observa-se através das médias que houve um aumento significativo do ganho de peso para codornas mantidas a $26^{\circ} \mathrm{C}$, o que corrobora com os valores de ITGU encontrados neste estudo. O maior ganho de peso se deu para as aves criadas no ambiente FMF1/CF2, com valor de $82,72 \mathrm{~g} /$ ave, demonstrando que estas aves conseguiram se manter no novo ambiente térmico. Ainda, dentro deste contexto, as codornas alojadas nos ambientes QMF1/CF2 QSF1/CF1, obtiveram consumo de ração de 65,30g/ave e 54,90g/ave, respectivamente. $\mathrm{Ou}$ seja, consumo abaixo daqueles encontrados para as aves mantidas na fase inicial de vida no frio moderado e severo, comprovando que, mesmo que estas aves tenham sido mantidas no conforto elas não conseguiram manter o desempenho.

Tabela 3. Médias e coeficientes de variações para o consumo de ração (CR), ganho de peso (GP), eficiência alimentar (EA) e viabilidade (VIAB) na criação das codornas de corte durante a quarta semana de vida (22 a 28 dias) em função dos diferentes ambientes térmicos adotados

\begin{tabular}{|c|c|c|c|c|}
\hline Ambientes Térmicos & CR (g/ave) & GP (g/ave) & EA & VIAB (\%) \\
\hline $\mathrm{CF} 1 / \mathrm{CF} 2$ & $191,25^{\mathrm{a}}$ & $77,10^{\mathrm{ab}}$ & $0,40^{\mathrm{a}}$ & $100,00^{\mathrm{a}}$ \\
\hline FMF1/CF2 & $209,08^{\mathrm{a}}$ & $82,72^{\mathrm{a}}$ & $0,36^{\mathrm{a}}$ & $95,00^{\mathrm{a}}$ \\
\hline FSF1/CF2 & $210,40^{\mathrm{a}}$ & $73,40^{\mathrm{ab}}$ & $0,35^{\mathrm{a}}$ & $100,00^{\mathrm{a}}$ \\
\hline QMF1/CF2 & $176,75^{\mathrm{a}}$ & $65,30^{\mathrm{ab}}$ & $0,37^{\mathrm{a}}$ & $100,00^{\mathrm{a}}$ \\
\hline QSF1/CF2 & $166,95^{\mathrm{a}}$ & $54,90^{\mathrm{ab}}$ & $0,33^{\mathrm{a}}$ & $100,00^{\mathrm{a}}$ \\
\hline CF1/QMF2 & $185,00^{\mathrm{a}}$ & $77,20^{\mathrm{ab}}$ & $0,42^{\mathrm{a}}$ & $100,00^{\mathrm{a}}$ \\
\hline FMF1/QMF2 & $180,80^{\mathrm{a}}$ & $60,90^{\mathrm{ab}}$ & $0,34^{\mathrm{a}}$ & $100,00^{\mathrm{a}}$ \\
\hline FSF1/QMF2 & $190,55^{\mathrm{a}}$ & $67,70^{\mathrm{ab}}$ & $0,36^{\mathrm{a}}$ & $100,00^{\mathrm{a}}$ \\
\hline QMF1/QMF2 & $206,00^{\mathrm{a}}$ & $72,90^{\mathrm{ab}}$ & $0,36^{\mathrm{a}}$ & $100,00^{\mathrm{a}}$ \\
\hline QSF1/QMF2 & $169,20^{\mathrm{a}}$ & $52,20^{\mathrm{ab}}$ & $0,31^{\mathrm{a}}$ & $100,00^{\mathrm{a}}$ \\
\hline CF1/QSF2 & $192,35^{\mathrm{a}}$ & $64,00^{\mathrm{ab}}$ & $0,33^{\mathrm{a}}$ & $100,00^{\mathrm{a}}$ \\
\hline FMF1/QSF2 & $160,30^{\mathrm{a}}$ & $72,90^{\mathrm{ab}}$ & $0,45^{\mathrm{a}}$ & $100,00^{\mathrm{a}}$ \\
\hline FSF1/QSF2 & $170,05^{\mathrm{a}}$ & $56,90^{\mathrm{ab}}$ & $0,33^{\mathrm{a}}$ & $100,00^{\mathrm{a}}$ \\
\hline QMF1/QSF2 & $166,55^{\mathrm{a}}$ & $70,80^{\mathrm{ab}}$ & $0,42^{\mathrm{a}}$ & $100,00^{\mathrm{a}}$ \\
\hline QSF1/QSF2 & $176,60^{\mathrm{a}}$ & $48,30^{\mathrm{b}}$ & $0,27^{\mathrm{a}}$ & $100,00^{\mathrm{a}}$ \\
\hline Coeficiente de Variação & 8,7 & 12,2 & 15,4 & 1,8 \\
\hline \multicolumn{5}{|c|}{$\begin{array}{l}\text { CF1/CF2 = Conforto Preconizado F1 + Conforto Preconizado F2 / FMF1/CF2 = Frio moderado F1+ } \\
\text { Conforto Preconizado F2 / FSF1/CF2 = Frio severo F1+ Conforto Preconizado F2 / QMF1/CF2 = Calor } \\
\text { moderado F1 + Conforto Preconizado F2 / QSF1/CF2 = Calor severo F1 + Conforto Preconizado F2 / } \\
\text { CF1/QMF2 = Conforto Preconizado F1+ Calor moderado F2 / FMF1/QMF2 = Frio moderado F1 + } \\
\text { Calor moderado F2 / FSF1/QMF2 = Frio severo F1+ Calor moderado F2 / QMF1/QMF2 = Calor } \\
\text { moderado F1 + Calor moderado F2 / QSF1/QMF2 = Calor severo F1 + Calor moderado F2 / CF1/QSF2 } \\
\text { = Conforto Preconizado F1+ Calor severo F2 / FMF1/QSF2 = Frio moderado F1 + Calor severo F2 / } \\
\text { FSF1/QSF2 = Frio severo F1 + Calor severo F2 / QMF1/QSF2 = Calor moderado F1+ Calor severo F2 / } \\
\text { QSF1/QSF2 = Calor severo F1 + Calor severo F2. } \\
\text { As médias seguidas com letras diferentes na coluna diferem entre sido ao nível de 5\% de probabilidade } \\
\text { pelo teste de Tukey. }\end{array}$} \\
\hline
\end{tabular}

Por outro lado, o pior ganho de peso, foi verificado para as aves que foram mantidas no QSF1/QSF2, com valor de 48,30g/ave, diferença em termos porcentuais de 41,61 a menos, quando comparado com o tratamento FMF1/CF2 
(82,72g/ave), e isto é um indicativo de que as codornas quando criadas durante o ciclo completo da sua vida, em temperaturas de desconforto térmico (principalmente no calor), têm o seu desempenho comprometido. Além disso, ao compararmos os tratamentos QMF1/QSF2 e QSF1/QSF2 (Tabela 3), percebe-se que houve maior ganho de peso para os animais mantidos no QMF1/QSF2. Diante disso, analisando que as respostas em termos de desempenho são diferentes, inferindo-se que as aves quando mantidas em estresse por calor mais ameno na sua fase inicial de vida, conseguem manter ou até mesmo melhorar seu desempenho produtivo, sendo melhor adaptadas ao novo ambiente. Este resultado sugere uma melhor aclimatação destas aves, quando comparadas com aquelas que, durante o ciclo completo de vida (inicial e final), foram mantidas a uma temperatura de calor severo crônico (como foi o caso do QSF1/QSF2). Em termos percentuais, as codornas expostas a estas condições obtiveram ganho de peso $31,78 \%$ menor, quando comparadas àquelas oriundas ao ambiente QMF1/QSF2.

De acordo com Cassuce et al. (2012), é importante colocar as aves em ambientes termicamente adequados desde os seus primeiros dias de vida, já que as mesmas não são capazes de se recuperar plenamente nas fases seguintes do seu período de criação.

Embora não tenham sido significativos, verificou-se que a maioria dos ambientes térmicos nos quais as codornas foram mantidas em temperaturas de conforto, proporcionaram maior consumo de ração por ave durante a quarta semana de vida, isto se deve ao menor valor de temperatura adotada $\left(26^{\circ} \mathrm{C}\right.$ - conforto preconizado) e ITGU $(75,8 \pm 0,9)$.

Sevegnani et al. (2005) avaliaram o comportamento de frangos de corte com diferentes idades submetidos a diversas combinações de temperatura e umidade em câmara climática. Os resultados mostraram que, em aves com idade mais avançada, à medida em que a temperatura se eleva, ocorre maior ingestão de água e menor ingestão de ração.

Grieser (2012), avaliando o crescimento e o desenvolvimento de codornas de corte encontrou valores de $185,12 \mathrm{~g} / \mathrm{ave} ; 50,21 \mathrm{~g} /$ ave e $163,55 \mathrm{~g} / \mathrm{ave}$, respectivamente para peso aos 22 dias, ganho de peso e consumo de ração, em relação ao mesmo período deste experimento. Já Otutumi et al. (2009) avaliando o desempenho de codornas de corte com adição de probióticos, verificou valores de 151,51g/ave e $48,79 \mathrm{~g} / \mathrm{ave}$, respectivamente para $\mathrm{o}$ consumo de ração e ganho de peso, de 22 a 28 dias.

Verificou-se efeito dos ambientes térmicos $(\mathrm{P} \leq 0,05)$, sobre $\mathrm{o}$ peso corporal das codornas aos 29 e 35 dias de idade. Ao contrário, observou-se que o consumo de ração, o ganho de peso, a eficiência alimentar e a viabilidade das aves não foram influenciadas $(\mathrm{P} \geq 0,05)$ pelos ambientes térmicos de criação adotados (Tabela 4).

Para o peso aos 29 dias, os valores observados em geral, foram maiores para as codornas mantidas no tratamento conforto preconizado durante a fase final de criação, o que estão de acordo com os valores encontrados de ITGU, pois foram os mais adequados em termos de conforto para o desempenho de codornas. Sendo que, o maior valor do peso vivo para esta semana foi para o tratamento FMF1/QMF2 com 204,10 $\mathrm{g} /$ ave, diante disso, entende-se que as aves tentaram recuperar seu desempenho, quando transferidas para uma temperatura de calor mais elevado. Por outro lado, o menor peso, se deu para o tratamento CF1/QSF2 com $171,60 \mathrm{~g} / \mathrm{ave}$, pois os valores de ITGU 
Rev. Bras. Saúde Prod. Anim., Salvador, v.15, n.2, p.350-360 abr./jun., 2014 http://www.rbspa.ufba.br ISSN 15199940

encontrados para esta semana estavam acima dos considerados como satisfatórios para o bom desempenho das aves, e ainda, os valores de ITGU estiveram mais altos quando comparados com a semana anterior.

Tabela 4. Médias e coeficientes de variações para o peso aos 29 dias de idade (P29 dias), peso aos 35 dias de idade (P35dias), consumo de ração (CR), ganho de peso (GP), eficiência alimentar (EA) e viabilidade (VIAB) na criação das codornas de corte durante a última semana de vida (29 a 35 dias) em função dos diferentes ambientes térmicos adotados

\begin{tabular}{|c|c|c|c|c|c|c|}
\hline Ambientes Térmicos & P29dias & P35dias & $\mathrm{CR}$ & GP & EA & VIAB \\
\hline $\mathrm{CF} 1 / \mathrm{CF} 2$ & $186,10^{\mathrm{ab}}$ & $230,50^{\mathrm{ab}}$ & $198,05^{\mathrm{a}}$ & $44,40^{\mathrm{a}}$ & $0,22^{\mathrm{a}}$ & $100,00^{\mathrm{a}}$ \\
\hline FMF1/CF2 & $200,02^{\mathrm{ab}}$ & $248,84^{\mathrm{a}}$ & $214,72^{\mathrm{a}}$ & $48,82^{\mathrm{a}}$ & $0,23^{\mathrm{a}}$ & $100,00^{\mathrm{a}}$ \\
\hline FSF1/CF2 & $201,60^{\mathrm{ab}}$ & $254,80^{\mathrm{a}}$ & $194,50^{\mathrm{a}}$ & $53,20^{\mathrm{a}}$ & $0,27^{\mathrm{a}}$ & $100,00^{\mathrm{a}}$ \\
\hline $\mathrm{QMF} 1 / \mathrm{CF} 2$ & $192,40^{\mathrm{ab}}$ & $250,60^{a}$ & $219,95^{\mathrm{a}}$ & $58,20^{\mathrm{a}}$ & $0,26^{\mathrm{a}}$ & $100,00^{\mathrm{a}}$ \\
\hline QSF1/CF2 & $182,00^{\mathrm{ab}}$ & $226,60^{\mathrm{ab}}$ & $180,10^{\mathrm{a}}$ & $44,60^{\mathrm{a}}$ & $0,25^{\mathrm{a}}$ & $100,00^{\mathrm{a}}$ \\
\hline CF1/QMF2 & $184,20^{\mathrm{ab}}$ & $238,99^{\mathrm{a}}$ & $192,65^{\mathrm{a}}$ & $54,78^{\mathrm{a}}$ & $0,23^{\mathrm{a}}$ & $100,00^{\mathrm{a}}$ \\
\hline FMF1/QMF2 & $204,10^{\mathrm{a}}$ & $248,60^{\mathrm{a}}$ & $195,75^{\mathrm{a}}$ & $44,50^{\mathrm{a}}$ & $0,23^{\mathrm{a}}$ & $100,00^{\mathrm{a}}$ \\
\hline FSF1/QMF2 & $196,60^{\mathrm{ab}}$ & $227,70^{\mathrm{ab}}$ & $181,25^{\mathrm{a}}$ & $31,10^{\mathrm{a}}$ & $0,17^{\mathrm{a}}$ & $100,00^{\mathrm{a}}$ \\
\hline QMF1/QMF2 & $178,80^{\mathrm{ab}}$ & $226,90^{\mathrm{ab}}$ & $194,20^{\mathrm{a}}$ & $48,10^{\mathrm{a}}$ & $0,25^{\mathrm{a}}$ & $100,00^{\mathrm{a}}$ \\
\hline QSF1/QMF2 & $178,40^{\mathrm{ab}}$ & $223,00^{\mathrm{ab}}$ & $166,25^{\mathrm{a}}$ & $44,60^{\mathrm{a}}$ & $0,27^{\mathrm{a}}$ & $100,00^{\mathrm{a}}$ \\
\hline CF1/QSF2 & $171,60^{\mathrm{b}}$ & $225,80^{\mathrm{ab}}$ & $157,95^{\mathrm{a}}$ & $54,20^{\mathrm{a}}$ & $0,34^{\mathrm{a}}$ & $100,00^{\mathrm{a}}$ \\
\hline FMF1/QSF2 & $188,30^{\mathrm{ab}}$ & $213,80^{\mathrm{b}}$ & $167,85^{\mathrm{a}}$ & $25,50^{\mathrm{a}}$ & $0,15^{\mathrm{a}}$ & $100,00^{\mathrm{a}}$ \\
\hline FSF1/QSF2 & $184,80^{\mathrm{ab}}$ & $232,90^{\mathrm{ab}}$ & $183,30^{\mathrm{a}}$ & $48,10^{\mathrm{a}}$ & $0,26^{\mathrm{a}}$ & $100,00^{\mathrm{a}}$ \\
\hline QMF1/QSF2 & $175,90^{\mathrm{ab}}$ & $215,98^{\mathrm{b}}$ & $164,06^{\mathrm{a}}$ & $40,07^{\mathrm{a}}$ & $0,27^{\mathrm{a}}$ & $90,00^{\mathrm{a}}$ \\
\hline QSF1/QSF2 & $174,80^{\mathrm{ab}}$ & $224,24^{\mathrm{ab}}$ & $184,66^{\mathrm{a}}$ & $49,44^{\mathrm{a}}$ & $0,29^{\mathrm{a}}$ & $85,00^{\mathrm{a}}$ \\
\hline Coeficiente de Variação & 4,3 & 4,6 & 12,3 & 20,1 & 19,0 & 6,7 \\
\hline
\end{tabular}

CF1/CF2 = Conforto Preconizado F1 + Conforto Preconizado F2 / FMF1/CF2 = Frio moderado F1+ Conforto Preconizado F2 / FSF1/CF2 = Frio severo F1+ Conforto Preconizado F2 / QMF1/CF2 = Calor moderado F1 + Conforto Preconizado F2 / QSF1/CF2 = Calor severo F1 + Conforto Preconizado F2 / CF1/QMF2: Conforto Preconizado F1+ Calor moderado F2 / FMF1/QMF2: Frio moderado F1 + Calor moderado F2 / FSF1/QMF2 = Frio severo F1+ Calor moderado F2 / QMF1/QMF2 = Calor moderado F1 + Calor moderado F2 / QSF1/QMF2 = Calor severo F1 + Calor moderado F2 / CF1/QSF2 = Conforto Preconizado F1+ Calor severo F2 / FMF1/QSF2 = Frio moderado F1 + Calor severo F2 / FSF1/QSF2 = Frio severo F1 + Calor severo F2 / QMF1/QSF2 = Calor moderado F1+ Calor severo F2 / QSF1/QSF2: Calor severo F1 + Calor severo F2.

As médias seguidas com letras diferentes na coluna diferem entre sido ao nível de $5 \%$ de probabilidade pelo teste de Tukey.

Assim, diante dos valores encontrados, percebe-se que as codornas que na fase inicial de vida foram alojadas em temperaturas tidas como de conforto, quando transferidas na fase final de criação e, alojadas em altas temperaturas, não mantiveram e nem recuperaram seu desempenho, o mesmo aconteceu para as codornas mantidas na fase inicial de vida em altas temperaturas (calor moderado e severo), e em seguida mantidas em temperatura de conforto.
Para o peso aos 35 dias, os valores também foram maiores para as codornas mantidas nos tratamentos conforto preconizado para segunda fase de criação $(\mathrm{P} \leq 0,05), \quad$ assim, isto se relaciona diretamente com os valores de ITGU encontrados para estes ambientes térmicos e para esta semana (75,3 \pm 0,7). O maior peso final (35 dias), embora não significativo foi para o tratamento FSF1/CF2 com 254,80g/ave. Desta forma, observou-se que as 
codornas deste tratamento também tentaram recuperar o desempenho, quando transferidas para a temperatura de conforto, pois tiveram ganho de peso de 53,20g/ave, relativamente mais alto quando comparados com os outros tratamentos. O menor peso $(\mathrm{P} \leq 0,05)$, se deu para o tratamento FMF1/QSF2 com $213,80 \mathrm{~g} / \mathrm{ave}$, com $16,10 \%$ a menos quando comparado com tratamento FSF1/CF2.

Embora para o consumo de ração não tenham sido encontradas diferenças significativas $(P \geq 0,05), \quad o \quad$ maior valor $(219,50 \mathrm{~g} / \mathrm{ave} /$ período $)$ se deu para o tratamento QMF1/CF2. Já o menor valor para este parâmetro (157,95g/ave/período), se deu no tratamento CF1/QSF2, com 28,04\% a menos quando comparado com $\mathrm{O}$ tratamento QMF1/CF2. Isto comprova, novamente, que as codornas quando mantidas na fase inicial de vida em temperaturas de conforto, quando transferidas para temperaturas de estresse por calor, têm seu desempenho piorado, o que não é desejado nas atuais condições comerciais das granjas avícolas.

Verificou-se que, a maioria das codornas mantidas nos tratamentos de conforto na fase final de criação, tiveram maior consumo voluntário de ração. Diante desta premissa, podemos afirmar que, quanto maior for a temperatura ambiental, menor será a ingestão voluntária de alimentos, pois o incremento calórico proveniente da ração, em altas temperaturas não é satisfatório, e a ave precisa eliminar este excesso de calor.

Diante do exposto, verificou-se que as codornas mantidas anteriormente (fase inicial de vida) em temperaturas de estresse por frio (moderado e severo), quando levadas para situações de conforto, melhoram o desempenho produtivo (ganho de peso), quando comparadas com aquelas que foram submetidas ao estresse por calor moderado e severo.

Vercese et al. (2012) avaliando o efeito do estresse contínuo pelo calor sobre o desempenho e a qualidade dos ovos de codornas japonesas, alojadas em câmaras climáticas, observou que a partir de $27^{\circ} \mathrm{C}$ de exposição às condições de estudo, as aves já apresentaram evidências de estresse térmico com redução no consumo de ração, peso e massa dos ovos. A elevação cíclica de $36^{\circ} \mathrm{C}$ na temperatura ambiente afetou negativamente a produção, comprovando o consequente efeito do estresse térmico no metabolismo e no equilíbrio térmico corporal das aves.

Mesmo não tendo sido encontradas diferenças significativas $(\mathrm{P} \geq 0,05)$ para $\mathrm{o}$ ganho de peso nesta última semana de vida das codornas, as aves mantidas no tratamento QMF1/CF2, obtiveram o maior ganho de peso quando comparado com os demais tratamentos $(58,20$ g/ave/período). Enquanto, que o menor ganho de peso se deu para o tratamento FMF1/QSF2, com ganho de 25,50g/ave, uma diferença em termos percentuais de 56,18 a menos, quando comparados com o tratamento QMF1/CF2.

Outros valores que podemos destacar, neste mesmo parâmetro (ganho de peso), foram para os ambientes térmicos QMF1/QSF2 e QSF1/QSF2, com valores de 40,07 e 49,44g/ave, respectivamente. Os animais que permaneceram durante o ciclo completo de produção (inicial e final) em temperaturas por calor severo e crônico, mantiveram seu desempenho produtivo, ou seja, estas codornas já estavam aclimatizadas ao ambiente térmico, quando comparada com o tratamento QMF1/QSF2, no qual, as mesmas tiveram que se adaptar a um novo ambiente, com temperaturas mais elevadas. Ao analisarmos esta diferença, verifica-se que as codornas do QSF1/QSF2, ganharam 18,95\% de peso 
a mais, quando comparadas com as codornas do QMF1/QSF2.

Otutumi et al. (2009), verificou valores para esta última semana da fase final, de $210,44 \mathrm{~g} /$ ave e $51,43 \mathrm{~g} / \mathrm{ave}$, respectivamente para o consumo de ração e o ganho de peso. Enquanto que, Grieser (2012), encontrou valores de $225,76 \mathrm{~g} / \mathrm{ave} ; 40,64 \mathrm{~g} /$ ave e $201,77 \mathrm{~g} / \mathrm{ave}$, respectivamente para o peso aos 35 dias, o ganho de peso e consumo de ração, em relação ao mesmo período deste experimento.

Embora não significativo $(\mathrm{P} \geq 0,05)$, para a viabilidade desta última semana experimental, pode-se dizer que houve baixa ou quase nenhuma mortalidade entre os tratamentos. Entretanto, as codornas mantidas nos tratamentos QMF1/QSF2 e QSF1/QSF2, ambas mantidas sob temperatura considerada como calor severo $\left(33^{\circ} \mathrm{C}\right)$, tiveram maior mortalidade, as aves não suportaram o calor crônico durante todo o ciclo de vida, quando comparadas com os demais tratamentos. Pode-se dizer que, codornas na fase final de criação, toleram menos ao calor do que ao frio, ao contrário das primeiras semanas de vida (fase de aquecimento).

Com base nos resultados de desempenho encontrados, a temperatura de conforto para codornas de corte durante a quarta e quinta semanas de vida destas são, $26^{\circ} \mathrm{C}$ e $25^{\circ} \mathrm{C}$, respectivamente. Acima destes valores já ocorrem perdas produtivas e, valores de ITGU entre 75,3 $\pm 0,7$ e 75,8 $\pm 0,9$ são considerados os ideais para a fase final de criação, sendo que, ITGU acima de 76,7 já ocorre queda no desempenho. Ainda, codornas criadas em condições de conforto térmico na fase inicial, quando submetidas (na fase final de vida) à temperaturas de estresse por calor seja moderado ou severo crônicos, não conseguem manter a performance normal, demonstrando necessidades de se garantir que o ambiente térmico dos aviários seja mantido adequado de acordo com as exigências de cada idade da ave.

\section{AGRADECIMENTOS}

Ao CNPq (Conselho Nacional de Pesquisa e Desenvolvimento), INCT (Instituto Nacional de Ciência e Tecnologia de Ciência Animal) pelo auxílio financeiro ao projeto executado e a Capes (Coordenação de Aperfeiçoamento de Pessoal de Nivel Superior) pela concessão da bolsa de estudos.

\section{REFERÊNCIAS}

ALBINO, L.F.T, BARRETO, S.L.T. Codornas: criação de codornas para produção de ovos e carne. Viçosa: Aprenda Fácil, 2003. 289p.

BAÊTA, F.C.; SOUZA, C.F. Ambiência em edificações rurais, conforto animal. Viçosa, MG: UFV, 2010. 269p.

BUFFINGTON, D.E.; COLLASSOAROCHO, A.; CANTON, G.H.; PITT, D. Black globehumidity index (BGTH) as comfort equation for dairy cows.

Transaction of the ASAE, v.24, n.3, p.711-714, 1981.

CASSUCE, D.C.; TINOCO, I.F.F.; BAETA, F.C.; ZOLNIER, S.; CECON, P.R.; VIEIRA, M.F.A. Atualização das temperaturas de conforto térmico para frangos de corte de até 21 dias de idade. Engenharia Agrícola (Online), v.33, p.28-36, 2012.

FURTADO, D.A.; DANTAS, R.T.; NASCIMENTO, J.W.B.; SANTOS, J.T.; COSTA, F.G.P. Efeitos de diferentes sistemas de acondicionamento ambiente sobre o desempenho produtivo de frangos de corte. Revista Brasileira de 
Rev. Bras. Saúde Prod. Anim., Salvador, v.15, n.2, p.350-360 abr./jun., 2014 http://www.rbspa.ufba.br ISSN 15199940

Engenharia Agrícola e Ambiental, v.10, n.2, p.484-489, 2006.

GRIESER, D.O. Estudo do crescimento e composição corporal de linhagens de codornas de corte e postura. 2012. 109p. Dissertação (Mestrado em Zootecnia) - Universidade Estadual de Maringá, Maringá.

MEDEIROS, C.M.; BAÊTA, F.C.; OLIVEIRA, R.F.M.; TINÔCO, I.F.F.; ALBINO, L. F.T.; CECON, P.R. Efeitos da temperatura, umidade relativa $\mathrm{e}$ velocidade do ar em frangos de corte.

Engenharia na Agricultura, v.13, n.4, p.277-286, 2005.

OLIVEIRA, R.F.M.; DONZELE, J.L.; ABREU, M.L.T.; FERREIRA, T.A.; VAZ, R.G.M.V.; CELLA, P.S. Efeitos da temperatura e da umidade relativa sobre $o$ desempenho e o rendimento de cortes nobres de frangos de corte de 1 a 49 dias de idade. Revista Brasileira de Zootecnia, v.35, n.3, p.797-803, 2006.

OTUTUMI, L.K.; FURLAN, A.C.; MARTINS, E.N.; GARCIA, E.R.M.; TON, A.P.S.; MONTEIRO, A.C. Efeito do probiótico sobre o desempenho, rendimento de carcaça e exigências nutricionais de proteína bruta de codornas de corte. Revista Brasileira de Zootecnia, v.38, n.2, p.299-306, 2009.

ROSTAGNO, H.S.; ALBINO, L.F.T.; DONZELE, J.L.; GOMES, P.C.; OLIVEIRA, R.F.M.; LOPES, D.C.; FERREIRA, A.S.; BARRETO, S.L.T.; EUCLIDES, R.F. Tabelas brasileiras para aves e suínos: composição de alimentos e exigências nutricionais. 3.ed. Viçosa, MG: UFV, 2011. 252p.

SEVEGNANI, K.B.; CARO, I.W.; PANDORFI, H.; SILVA, I.J.O.; MOURA, D.J. Zootecnia de precisão: análise de imagens no estudo do comportamento de frangos de corte em estresse térmico. Revista Brasileira de Engenharia Agrícola e Ambiental, v.9, n.1, p.115-119, 2005.

SILVA, J.H.V; COSTA, F.G.P. Tabelas para codornas japonesas e européias. 2.ed. Jaboticabal, SP: Funep, 2009. 107p.

SILVA, J.H.V.; JORDÃO FILHO, J.; COSTA, F.G.P.; LACERDA, P.B.; VARGAS, D.G.V.; LIMA, M.R. Exigências nutricionais de codornas. Revista Brasileira de Saúde e Produção Animal [online], v.13, n.3, p.775-790, 2012.

TEIXEIRA, V.H. Estudos dos índices de conforto em duas instalações de frango de corte para as regiões de Viçosa e Visconde do Rio Branco, MG. 1983. 62p. Dissertação (Mestrado em Engenharia Agrícola) - Universidade Federal de Viçosa, Viçosa.

UNIVERSIDADE FEDERAL DE VIÇOSA - UFV. SAEG - Sistema para análise estatística e genética. Versão 9,1. Viçosa, MG, 2007.

VAZ, R.G.M.V.; OLIVEIRA, R.F.M.; DONZELE, J.L.; ALBINO, L.F.T.; OLIVEIRA, W.P.; SILVA, B.A.N. Inclusão de cromo orgânico em rações para frangos de corte mantidos sob estresse por calor no período de um a 42 dias de idade. Arquivo Brasileiro de Medicina Veterinária e Zootecnia, v. 61, n.2, p.484-490, 2009.

VERCESE, F.; GARCIA, E. A.; SARTORI, J. R.; SILVA, A.P.; FAITARONE, A.B.G.; BERTO, D.; BRITO, A.M.; PELICIA, K. Performance and egg quality of Japanese quails submitted to cyclic heat stress. Revista Brasileira de Ciência Avícola, v.14, p.37-41, 2012.

Data de recebimento: 08/07/2013

Data de aprovação: 02/06/2014 